\title{
Stress-induced release of matrix metalloproteinase-9 in patients with coronary artery disease: The possible influence of cortisol
}

\author{
Anna Lundberg, Simon Jönsson, Jonathan Stenmark, Margareta Kristenson and Lena \\ Jonasson \\ Journal Article
}

\section{Tweet}

N.B.: When citing this work, cite the original article.

Original Publication:

Anna Lundberg, Simon Jönsson, Jonathan Stenmark, Margareta Kristenson and Lena Jonasson, Stress-induced release of matrix metalloproteinase- 9 in patients with coronary artery disease: The possible influence of cortisol, Psychoneuroendocrinology, 2016. 73(), pp.117-124.

http://dx.doi.org/10.1016/j.psyneuen.2016.07.219

Copyright: Elsevier

http://www.elsevier.com/

Postprint available at: Linköping University Electronic Press

http://urn.kb.se/resolve?urn=urn:nbn:se:liu:diva-132516

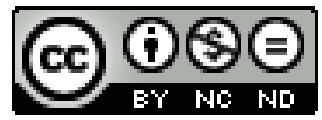


Stress-induced release of matrix metalloproteinase- 9 in patients with coronary artery disease: The possible influence of cortisol.

Anna K Lundberga ${ }^{a^{*}}$, Simon Jönsson ${ }^{a^{*}}$, Jonathan Stenmark ${ }^{a}$, Margareta Kristenson ${ }^{b}$, Lena Jonasson ${ }^{a}$

aDivision of Cardiovascular Medicine, Department of Medical and Health Sciences, Faculty of Health Sciences, Linköping University, Linköping, Sweden,

${ }^{\mathrm{b}}$ Division of Community Medicine, Department of Medical and Health Sciences, Division of Community Medicine, Linköping University, Sweden.

*contributed equally

Correspondence: Lena Jonasson, Division of Cardiovascular Medicine, Department of Medical and Health Sciences, Faculty of Health Sciences, Linköping University, SE-58185 Linköping, Sweden. lena.jonasson@liu.se 


\section{Abstract}

Stress and inflammation are both important risk factors for coronary artery disease (CAD). However, the susceptibility to stress-induced inflammation and its determinants have been little explored in patients with CAD. Here, our aim was to study the stress-induced inflammatory response, more precisely the early release of matrix metalloproteinase (MMP)-9, and its association with cortisol response in patients with CAD. Sixty-four patients underwent a standardized laboratory stress test. The stress-induced release of MMP-9 was closely associated with the release of other neutrophilassociated proteins, MMP-8 and myeloperoxidase (MPO). It also showed a large variation among patients, as did cortisol. Twenty minutes after stress, a negative association between changes in MMP-9 and cortisol was seen $(\mathrm{p}<0.01)$. In vitro, dexamethasone reduced the IL-8-mediated release of MMP-9 from neutrophils, indicating that glucocorticoids may exert rapid effects on neutrophil activation. Further characterization of patients revealed that stress-induced release of MMP-9 was related to leukocyte telomere shortening and increased ultrasound-assessed plaque occurrence in the carotid arteries, but not to other characteristics such as age, gender or psychological background factors. The susceptibility to stress-induced release of MMP-9 may thus have impact on disease phenotype. Stress tests can be useful to identify CAD patients in need of novel prevention and treatment strategies.

\section{Key words}

Coronary artery disease; stress; inflammation; cortisol; matrix metalloproteinase; neutrophil 


\section{Introduction}

The association between psychological stress and cardiovascular disease has been documented in a number of epidemiological, clinical and experimental studies, as recently reviewed by Lagraauw et al (Lagraauw et al. 2015). Bouts of psychological stress also trigger cardiac events and this effect may be stronger in individuals with unfavorable psychological attributes, such as low coping skills and depressive mood (Edmondson et al. 2013). Still, it is unclear why some individuals are more susceptible to emotional stress than others and many questions remain to be answered about the mechanisms by which stress influences the cardiovascular risk. Inflammation has been proposed as one mechanism linking stress to increased risk of cardiovascular disease.

\subsection{Matrix metalloproteinase (MMP)-9 in coronary artery disease}

Atherosclerosis is a chronic inflammatory process in the arterial wall (Silvestre-Roig et al. 2014). As a mediator of inflammation and matrix-degrading protease, matrix metalloproteinase (MMP)-9 is considered to play a key role in both development of atherosclerosis and plaque destabilization. Its proatherogenic effects are well documented in experimental studies (Ketelhuth and Back 2011, Newby 2015). Circulating levels of MMP-9 have also been shown to predict cardiovascular risk (Blankenberg et al. 2003, Garvin et al. 2015). Moreover, independent associations between MMP-9 levels and psychological risk factors, such as depression, cynical hostility and coping, have been reported (Garvin et al. 2009, Domenici et al. 2010).

MMP-9 is expressed by a number of cell types in diverse tissues, including leukocytes, macrophages, mast cells and endothelial cells. In the circulation, neutrophils are considered to be the major source (Jönsson et al. 2011, Newby 2015). The enzyme is stored in a latent form in gelatinase-rich (or tertiary) granules of neutrophils and rapidly released following stimulation (Soehnlein 2012). In a previous study, we found that neutrophils in CAD patients were more prone to release MMP-9 ex vivo than were neutrophils from healthy controls. We also found that blood mononuclear cells in 
CAD patients had higher levels of MMP-9 mRNA compared with controls but that the secretion of MMP-9 ex vivo from mononuclear cells was negligible in both patients and controls (Jönsson et al. 2011).

\subsection{Stress-induced inflammatory response is counteracted by glucocorticoids}

Several studies have consistently shown that stressful stimuli can trigger an inflammatory response (Steptoe et al. 2007, Rohleder 2014, Kuebler et al. 2015). In order to maintain homeostasis, the hypothalamus-pituitary-adrenal (HPA) axis and its end product glucocorticoids (mostly cortisol in humans) have an essential role in counteracting stress-induced inflammatory response. An impaired glucocorticoid response may thus result in a failure to dampen the inflammatory response. Accordingly, it has been shown that the cortisol response is negatively associated with both rapid NF$\kappa \mathrm{B}$ activation in blood mononuclear cells and delayed increases in plasma cytokine levels when individuals are exposed to laboratory stress (Kunz-Ebrecht et al. 2003, Wolf et al. 2009, Brydon 2010). In a previous study, we found that blunted cortisol response to acute psychological stress was related to elevations in C-reactive protein and MMP-9 levels the day after stress in both CAD patients and healthy controls. The attenuation of cortisol response was more frequently observed in patients than in controls (Nijm et al. 2007, Szymanowski et al. 2011). Interestingly, a blunted cortisol response to stress has also been associated with shorter leukocyte telomere length (Tomiyama et al. 2012), the latter considered a marker of cardiovascular aging (Fyhrquist et al. 2013).

\subsection{Glucocorticoid effects on neutrophil activation}

Not only blood mononuclear cells are activated in response to stress. Stressful stimuli also induce activation of neutrophils (Ellard et al. 2001, Mian et al. 2003, Keresztes 2013). The result is a rapid release of granule proteins but also de novo synthesis of cytokines by neutrophils themselves leading to amplification of the inflammatory response. Glucocorticoids have been shown to inhibit cytokine gene expression in neutrophils through binding and activation of cytoplasmic glucocorticoid 
receptors (Hirsch et al. 2012). Whether glucocorticoids also inhibit the release of preformed granule proteins is less clear. In the present study, our main hypothesis was that an early stress-induced elevation in MMP-9 was associated with a blunted cortisol response to stress in CAD patients. Speculating that this might identify a potential high-risk disease phenotype, we also investigated whether stress-induced release of MMP-9 in CAD patients was associated with psychological risk factors, leukocyte telomere attrition and atherosclerotic burden.

\section{Methods}

\subsection{Study population}

Sixty-four patients with a recent myocardial infarction and/or a coronary revascularization procedure (percutaneous coronary intervention or coronary artery bypass graft surgery) as index event were consecutively recruited from the Outpatient Cardiology Clinic at the University Hospital in Linköping, Sweden, 4 weeks after the index event. Exclusion criteria were age $>75$ years, severe heart failure, neoplastic disease, major clinical depression, chronic immunologic disorders or treatment with immunosuppressive/anti-inflammatory agents. Psychometric measurements were performed on 3 separate occasions; 4 weeks, 12 months and 18 months after the index coronary event. Depressive symptoms and cynical hostility were assessed by using the Center for Epidemiological Studies Depression scale (CES-D) (Radloff 1977) and the 12-item Cook-Medley Hostility Scale (Cook and Medley 1954), respectively. Coping ability was measured by the Pearlin Mastery Scale (Pearlin 1978).

The study was conducted in accordance with the ethical guidelines of Declaration of Helsinki, and the research protocol was approved by the Ethical Review Board of Linköping University. Written informed consent was obtained from all study participants.

\subsection{Standardized laboratory stress test}


Between 6-12 months after the index event, all patients performed a standardized laboratory stress test. This test has been shown to elicit significant increases in heart rate and blood pressure, as described in our previous studies (Kristenson et al. 2005, Nijm et al. 2007). Instructed not to withhold their morning dose of cardiac medication, patients arrived to the laboratory at $8.00 \mathrm{pm}$ and after preparations including a relaxation phase, 2 different psychological stressors were presented in a fixed sequence with a 2-min interval to allow time for instructions. The rationale for combining 2 stressors was to increase the likelihood of a stress response since hemodynamic reactions to single stressors can vary between individuals. The first stressor was an "anger recall" when the patient was instructed to recall an event that made him/her angry, frustrated, or upset and had 6 min in which to relate what had happened and how he/she had felt. The second stressor was a mental arithmetic test. The patient was then instructed to count backwards, 700 minus 7 as quickly and correctly as possible and to try to reach zero within $4 \mathrm{~min}$. Blood pressure and heart rate were recorded before the first stressor and every 2 min for 34 minutes. Blood and saliva samples were collected directly before the first stressor and after $34 \mathrm{~min}$, i.e. $20 \mathrm{~min}$ after the conclusion of the second stressor.

\subsection{Analysis of MMP-9, MMP-8, myeloperoxidase (MPO) and interleukin (IL)-6}

Serum levels of MMP-9 and MMP-8 were measured by Luminex Performance Human MMP Panel, serum levels of MPO by Luminex Performance Human Cardiac Panel B, and EDTA-plasma levels of IL6 by QuantiGlo ELISA kit (all from R\&D Systems, Minneapolis MN, USA) according to the protocol provided by the manufacturer. The interassay percentage coefficients of variation were for MMP9: $13.8 \%$, MMP-8: $6.8 \%$, MPO: $5.0 \%$, and IL-6: $7.0 \%$. The minimum detectable doses (mean, $\mathrm{pg} / \mathrm{mL}$ ) were for MMP-9: 5.7, MMP-8: 7.8, MPO: 18.4, and IL-6: 0.16.

\subsection{Analysis of cortisol}

Saliva was collected with Salivette cotton swabs (Sarstedt, Nümbrecht, Germany) that were placed under the tongue for 2 minutes. The Salivettes were immediately frozen at $-20 \mathrm{C}^{\circ}$. 
The levels of free cortisol in saliva were determined at the accredited Clinical Chemical Laboratory at Karolinska University Hospital, Sweden by a commercial radioimmunoassay assay, CORT-CT2 (Cisbio Bioanalyser, Codolet, France). According to repeatedly performed quality assessments, the interassay coefficient of variance was less than $10 \%$. The limit of detection is $3.0 \mathrm{nmol} / \mathrm{L}$ and the limit of quantitation $100 \mathrm{nmol} / \mathrm{L}$.

\subsection{Telomere length measurement assay}

DNA was prepared from whole blood using Maxwell 16 blood DNA purification kit (Promega Biotech, Stockholm, Sweden) and sent to the Blackburn laboratory at the University of California, San Francisco, where telomere length assays were conducted, as previously described (Lin et al. 2010). The telomere length is measured as the ratio $(T / S)$ between the telomere product $(T)$ divided by single copy gene (S). The single copy gene measurement is to normalize for the quantity of DNA input. The ratio of $\mathrm{T} / \mathrm{S}$ reflects the length of the telomeres, since the longer the telomere is, the higher is the telomere product. Briefly, the primers used for the telomere PCR are tel1b [5'CGGTTT(GTTTGG)5GTT-3'] (final concentration of $100 \mathrm{nM}$ ), and tel2b [5'-GGCTTG(CCTTAC)5CCT-3'] (final concentration of $900 \mathrm{nM}$. The primers for the single-copy gene (human beta-globin) PCR are hbg1 [5' GCTTCTGACACAACTGTGTTCACTAGC-3'] (final concentration of 300 nM), and hbg2 [5'CACCAACTTCATCCACGTTCACC-3'] (final concentration of 700 nM). Reference DNA from Hela cancer cells were included in each PCR run and the quantity of targeted templates in each research sample was determined relative to the reference DNA sample by the standard curve method. The same reference DNA was used for all PCR runs.

\subsection{Carotid B-mode ultrasound}

Carotid intima-media thickness (IMT) and plaque occurrence were assessed in all patients between 6-12 months after the index event by B-mode ultrasound using a 9-18 Mhz linear 2D transducer (ACUSON S2000 TM ultrasound system, Siemens Medical Solutions USA, Inc.). IMT was measured in 
the common carotid artery $1 \mathrm{~cm}$ below the bifurcation of the external and internal carotid arteries. A mean value of IMT was determined by performing two repeated measurements. The occurrence of plaques was assessed by scanning the carotid arteries transversely and longitudinally. Plaques were defined according to the Mannheim Consensus (Touboul et al. 2012) and defined as structures $50 \%$ thicker than IMT in the focal area or a thickness $>1.5 \mathrm{~mm}$.

\subsection{In vitro neutrophil glucocorticoid suppression test}

In a separate substudy, fresh heparinised whole blood was drawn from 5 CAD patients and 5 healthy volunteers. Neutrophil isolation was performed using Polymorphprep (Axis-Shield PoC AS, Oslo, Norway) as previously described (Jönsson et al. 2011). Cell purity was assessed by microscopic examination. After isolation, the neutrophils were resuspended in RPMI-1640 with L-glutamine, 10\% FBS and $100 \mathrm{U} / \mathrm{ml}$ penicillin $100 \mu \mathrm{g} / \mathrm{ml}$ streptomycin to a concentration of $5 \times 10^{6}$ cells $/ \mathrm{ml}$. Cells $(1 \mathrm{~mL})$ were stimulated with $10 \mathrm{ng} / \mathrm{mL}$ of IL-8 (Sigma -Aldrich Corporation, St Louis, MO, USA) for $30 \mathrm{~min}$ in a $37^{\circ} \mathrm{C}$ water bath. To assess the inhibitory effects of glucocorticoids, neutrophils were preincubated with dexamethasone (Sigma-Aldrich Sweden $A B$ ) at various concentrations $\left(10^{-6} \mathrm{M}, 10^{-7} \mathrm{M}, 10^{-8} \mathrm{M}\right)$ for $5 \mathrm{~min}$. Thereafter, IL-8 was added and cells were further incubated for $30 \mathrm{~min}$. Finally, the cell suspensions were collected and centrifuged for $300 \times \mathrm{g}, 5 \mathrm{~min}, \mathrm{RT}$ and supernatants were collected for MMP-9, MMP-8 and MPO analysis by Luminex, as described above.

\subsection{Statistics}

Values are presented as median (inter-quartile range). Wilcoxon signed-ranks test was used for pairwise comparisons and Friedman's test was used to compare more than two observations repeated on the same subjects. Associations among variables were studied by means of the Pearson's correlation analysis of log-transformed data. Differences between groups were analyzed with Kruskal-Wallis test, and Mann-Whitney U-test was used for between-group analyses. A p-value $<0.05$ was considered statistically significant. IBM SPSS Statistics 21 was used for statistical analyses. 
Based on previous data on MMP-9 measurements performed before and $24 \mathrm{~h}$ after stress

(Szymanowski et al. 2011), we estimated that we would need to include 62 patients to detect a difference of $93 \mathrm{ng} / \mathrm{mL}$ in serum MMP-9 between cortisol non-responders (less than $10 \%$ increase in cortisol 30 minutes after stress) and cortisol responders in order to provide a power of $80 \%$ and a significance level of 0.05 .

\section{Results}

\subsection{Patients' characteristics and the cardiovascular response to stress}

Demographical, clinical and laboratory characteristics, cardiovascular medication and results from psychological and carotid ultrasound measurements are summarized in Table 1. CES-D scores did not vary significantly over time (Friedman's test, $p=0.68$ ), neither did cynical hostility or mastery scores ( $p=0.76$ and 0.39 , respectively). Therefore, arithmetic means of samples from the 3 test occasions are presented (and also used in all statistical analyses). The cardiovascular response to the laboratory stress test is shown in Table 2. Heart rate and systolic and diastolic blood pressures increased significantly already after the first stressor, and returned to baseline levels during recovery.

\subsection{Correlations between stress-induced changes in MMP-9, MMP-8, MPO and cortisol}

The release of MMP-9 in response to psychological stress showed a large variation and, therefore, a comparison between baseline and post-stress levels in the whole study sample revealed no significant difference, 311 (237-426) vs 315 (246-435) ng/mL. We also measured other neutrophilassociated proteins, namely MMP-8 (or neutrophil collagenase) and MPO, and found no differences between baseline and post-stress levels, $8.4(5.4-13)$ vs $9.0(6.0-13) \mathrm{ng} / \mathrm{mL}$, and 195 (158-299) vs 212 (160-294) ng/mL, respectively. Also, the cortisol response to stress varied substantially and overall, there were no significant differences between baseline and post-stress levels of salivary cortisol, 31 (23-39) vs 27 (21-37) nmol/L. The correlations between log-transformed levels of MMP-9, MMP-8, 
MPO and cortisol (including baseline levels and percentage changes after stress) are shown in Table

3. The baseline levels of MMP-9, MMP-8 and MPO correlated with each other and also with baseline neutrophil counts whereas baseline cortisol levels did not correlate with any neutrophil variables at baseline. After stress, the percentage changes in MMP-9, MMP-8 and MPO were strongly intercorrelated. All showed significant negative correlations with the percentage change in cortisol. The correlation between stress-induced changes in MMP-9 and cortisol is further illustrated in Figure 1.

We further assessed whether correlations persisted when study population was limited to males ( $\mathrm{n}=$ 54) and found that the change in cortisol still correlated with changes in MMP-9 $(r=-0.32, p=0.022)$ and MPO $(r=-0.35, p=0.011)$. When study population was limited to patients without diabetes $(n=$ $53)$, the change in cortisol still correlated with changes in MMP-9 $(r=-0.41, \mathrm{p}=0.002), \mathrm{MMP}-8(r=-$ $0.32, \mathrm{p}=0.021)$ and MPO $(r=-0.43, \mathrm{p}=0.002)$.

\subsection{Other correlates of stress-induced change in MMP-9}

The stress-induced release of MMP-9 did not show any correlations with heart rate or blood pressure responses. Neither did it correlate with psychological background factors (depressive symptoms, cynical hostility, coping ability) nor with other clinical variables, such as age, gender, BMI, smoking, diabetes or medication (data not shown). The stress-induced increase in MMP-9 correlated with baseline levels of IL-6, $r=0.29, \mathrm{p}=0.025$. It also correlated negatively with leukocyte telomere length, $r=-0.30, p=0.019$, and positively with presence of plaques (on the right side), $r=0.27, p=$ 0.034 , though not with other carotid ultrasound variables. Stress-induced changes in MMP-8 and MPO did not show any correlations with markers mentioned above.

\subsection{Patients divided into tertiles according to stress-induced change in MMP-9}

As an alternative way to present data, we divided the CAD patients into tertiles depending on their percentage change in MMP-9 (Figure 2). Selected characteristics of the tertile groups are shown in 
Table 4. The percentage changes in MMP-8 and MPO differed significantly across tertiles. Also, the cortisol response in the upper tertile (T3) differed significantly from the cortisol response in T1 and T2. Moreover, T3 exhibited a significant decrease in cortisol after stress (within-group, $p<0.001$ ), while T1 and T2 did not (within-group, $\mathrm{p}=0.81$ and 0.18 , respectively). Moreover, T3 exhibited higher baseline levels of IL-6 and shorter leukocyte telomere length compared with T1 and T2. The psychological background factors were similar across tertiles. Also, the cardiovascular reactivity to stress was similar (Table 4). There were no significant differences in age, gender, BMI, diabetes, smoking, cardiac medication (e.g. beta-blockers), neutrophil counts or base-line cortisol levels, neither were there any differences in IMT values or presence of plaques on the left side between tertiles (data not shown). However, T3 exhibited a significantly higher presence of plaques on the right side compared with $\mathrm{T} 1,100 \%$ vs $68 \%, \mathrm{p}=0.004$.

\subsection{Effect of dexamethasone on neutrophil release of MMP-9, MMP-8 and MPO}

In order to evaluate whether glucocorticoids exerted rapid inhibitory actions on human neutrophils, we assessed the effect of a synthetic glucocorticoid, dexamethasone, on isolated neutrophils from 10 individuals ( 5 CAD patients, 5 healthy controls). IL-8 was used to stimulate the release of MMP-9 from tertiary granules (Chakrabarti and Patel 2005). The release was significantly reduced by dexamethasone at various concentrations from $10^{-6}$ to $10^{-8} \mathrm{M}$ (Figure $3 \mathrm{~A}$ ). Also, the IL-8-induced release of MMP-8 was slightly reduced by dexamethasone (Figure 2B). The glucocorticoid effects were similar in patients and healthy volunteers. Treatment with dexamethasone without IL-8 did not affect the release of MMP-9 or MMP-8 (data not shown). Neither IL-8 nor dexamethasone affected the release of MPO into the supernatant (data not shown).

\section{Discussion}

\subsection{Large variation in stress-induced release of MMP-9 among CAD patients}


Acute stress giving rise to enhanced inflammatory response can be one major mechanism through which stress is linked to cardiovascular risk. Due to the release of preformed protein from neutrophils, MMP-9 can be detected in the circulation early after an inflammatory stimulus. It may therefore serve as as a useful marker of inflammation during stress. To our knowledge, this study is the first to investigate the early release of MMP-9 in response to a laboratory stress test. We found that the variation in stress-induced release of MMP-9 was substantial indicating that some CAD patients were vulnerable to stress-induced inflammation whereas others were not. In line with this, previous studies have shown that stress-induced release of other inflammatory markers, such as IL-6, varies markedly among healthy subjects (Steptoe et al. 2007, Wolf et al. 2009). Also, a variation in stress-induced inflammatory response among CAD patients has been illustrated by Strike et al (Strike et al. 2006) who divided patients into two groups: those who reported that their previous cardiac event had been preceded by negative emotional stress and those who did not report any emotional trigger. In response to mental stress, the emotion trigger group showed a marked increase in platelet activation while no such changes were seen in the non-trigger group. Strike et al (Strike et al. 2006) also reported increased levels of neutrophil-platelet aggregates in the trigger group suggesting the occurrence of stress-induced neutrophil activation.

We also measured circulating levels of MPO which is abundantly expressed in primary granules of neutrophils, and MMP-8 which is expressed in secondary and also, to a lesser extent, in tertiary granules (Soehnlein 2012). The increase in MPO and MMP-8 following stress strongly correlated with the increase in MMP-9, further supporting the presence of stress-activated neutrophils.

\subsection{Negative correlations between stress-induced changes in neutrophil-associated proteins and cortisol}

The cortisol response to stress also varied markedly among CAD patients. Twenty min after stress, as many as two-thirds of the study participants exhibited cortisol levels that were lower than pre-test levels. According to previous studies, a peak response in cortisol is expected to occur 15-25 min after 
a laboratory mental stressor followed by a rapid decline reaching below pre-test levels (Kunz-Ebrecht et al. 2003, Brydon 2010, McInnis et al. 2015). However, in one study of healthy volunteers, cortisol levels were shown to be lower already 20 min after stress compared with pre-test levels (Steptoe et al. 2001). Interestingly, Kunz-Ebrecht et al (Kunz-Ebrecht et al. 2003) reported that cortisol levels 20 min after stress were lower than pre-test levels in those individuals who exhibited the lowest cortisol response to stress, so-called cortisol non-responders. These authors also demonstrated that the cortisol stress response was negatively associated with proinflammatory cytokines suggesting that cortisol had an impact on the stress-induced inflammatory response. Subsequent studies further supported this theory by showing that cortisol response after laboratory stress correlated negatively with NF-KB activity in blood mononuclear cells (Wolf et al. 2009) and with plasma levels of IL-6 (Brydon 2010). In a recent study by Keresztes et al (Keresztes 2013), the association between HPA axis response and neutrophil activation was investigated in CAD patients undergoing percutaneous coronary intervention. A negative correlation between adrenocorticotropic hormone (ACTH) and lactoferrin, present in secondary neutrophil granules, was found the day after intervention suggesting a possible suppressive role of ACTH on neutrophil degranulation. In the present study, we observed significant negative correlations between stress-induced changes in cortisol and neutrophilassociated proteins after 20 minutes. This was further illustrated by dividing the patients into tertiles depending on their stress-induced changes in serum MMP-9. Cortisol declined significantly in the upper tertile only. Speculatively, this group of CAD patients may represent a group of cortisol nonresponders who fail to modulate their stress-induced neutrophil response.

\subsection{Dexamethasone-induced suppression of MMP-9 release from neutrophils}

We found that the IL-8-mediated release of MMP-9 from neutrophils in vitro was rapidly suppressed by dexamethasone. There were no apparent differences in glucocorticoid sensitivity between neutrophils from CAD patients and healthy subjects. However, the in vitro study was designed as a functional test only including cells from 10 individuals, thus not allowing the comparison between 
patients and controls. Also, the short-term stimulation with IL-8 was chosen to induce the release of MMP-9 from tertiary granules (Chakrabarti and Patel 2005). This may explain the weaker suppression of dexamethasone on MMP-8 secretion as well as the lack of effect on MPO secretion. Each granule type differs in how quickly their contents can be released, for example primary granules are the last to undergo exocytosis.

Glucocorticoids are known to exert delayed (or genomic) effects on neutrophils (Hirsch et al. 2012) but whether glucocorticoids also exert rapid (or non-genomic) effects on neutrophil priming have been less clear. In vitro studies on human neutrophils have shown contradictory results; in one study, treatment with dexamethasone $\left(10^{-6} \mathrm{M}\right)$ for $24 \mathrm{~h}$ failed to inhibit degranulation (Schleimer et al. 1989), while megadoses of $6 \beta$-methylprednisolone or hydrocortisone inhibited degranulation already after 10 min incubation in another study (Liu et al. 2005). Furthermore, in a more recent study using a human skin blister model of acute inflammation, the inhibitory effect of methylprednisolone was due to inhibition of neutrophil degranulation (Hellberg et al. 2013).

\subsection{Stress-induced release of MMP-9 - relation to psychological background factors}

We found no evidence that psychological factors, such as depressive symptoms (CES-D), cynical hostility or coping ability, influenced the stress-induced release of MMP-9. These psychological factors were chosen because they have shown independent associations with circulating MMP-9 levels in a community-based Swedish population (Garvin et al. 2009). Furthermore, stress-induced inflammatory response has shown association with both hostility and depressive symptoms. In a study of 76 patients with acute coronary syndrome, MMP-9 levels were associated with IL-1 $\beta$ levels but only in those with high hostility (Fernandez Machulsky et al. 2016). In another study of 34 patients with stable CAD, elevated IL-6 levels after laboratory stress were only detected in those with higher hostility scores (Brydon 2010). Moreover, when 138 healthy subjects were exposed to stress a greater IL-6 response was only seen in those with CES-D scores > 10 (Fagundes et al. 2013). In our study, the number of patients with high CES-D scores may be too few (27 patients with CES-D > 10) 
to draw any conclusions about depressive mood and stress-induced release of MMP-9. On the other hand, the repeated measurements of psychological background factors can be considered a strength. It should also be noted that large prospective studies have not been able to verify that inflammation is a link between depression and CAD (Nabi et al. 2008, Davidson et al. 2009). It is possible that the capacity to down-regulate the response to acute stressors is more important than psychological background factors in determining the susceptibility to stress-induced inflammation.

\subsection{Stress-induced release of MMP-9 - relation to leukocyte telomere attrition}

One interesting observation was that stress-induced release of MMP-9 correlated negatively with leukocyte telomere length. Telomere shortening is considered to be a marker of cardiovascular aging (Fyhrquist et al. 2013). Moreover, telomere shortening in leukocytes or blood mononuclear cells has shown associations with both blunted cortisol reactivity (Tomiyama et al. 2012) and hypocortisolism (Wikgren et al. 2012). Granulocytes, of which $>95 \%$ are neutrophils, represent the most abundant leukocytes in blood. Speculatively, short leukocyte telomeres may at least partly reflect a state of repetitive stimulation of granulocyte progenitor cells in the bone marrow. It was recently shown by Heidt et al (Heidt et al. 2014) that psychological stress increased proliferation of hematopoietic stem cells in the bone marrow of both wild-type and atherosclerosis-prone mice, resulting in increased output of neutrophils. Interestingly, the stressed atherosclerosis-prone mice exhibited a more inflammatory plaque phenotype involving higher neutrophil content and increased expression of MPO.

\subsection{Stress-induced release of MMP-9 - relation to carotid atherosclerosis}

Since coronary angiography is not an accurate method to assess atherosclerosis, carotid ultrasound was used to obtain an estimate of atherosclerotic burden. There was a weak but significant association between stress-induced release of MMP- 9 and presence of plaques. Although data should be interpreted with great caution, they are in line with previous studies indicating a key role 
for neutrophils in the development of disease (Soehnlein 2012, Della Bona et al. 2013). In a large human cohort, lonita et al (Ionita et al. 2010) demonstrated that high neutrophil numbers within the plaque correlated both with high intra-plaque levels of MMP-9 and MMP-8 and histopathologic features of rupture-prone lesions.

\subsection{Limitations}

Most important is to emphasize the limitations of our study, including the small sample size as well as the cross-sectional study design. The HPA axis response is complex and controlled by multiple factors. Although salivary cortisol is considered the measure of choice, measurements of ACTH and total cortisol in blood might have added useful information (Hellhammer et al. 2009). We believe that the early stress-induced increase in MMP-9, MMP-8 and MPO mainly reflects neutrophil activation. However, it would have been valuable to confirm the neutrophil activation status by e.g. flow cytometry. The lack of a healthy control group is another limitation. However, cardiovascular drugs may attenuate the cardiovascular response to stress and some of the most common drugs, e.g. statins and aspirin, have the potential to inhibit gene expression and release of MMP-9 (Bellosta et al. 1998, Hua et al. 2009). Thus, it would have been difficult to interpret whether a difference in stress-induced release of MMP-9 between medicated CAD patients and unmedicated controls represented the effect of CAD itself or the effect of medication.

\section{Conclusion}

To summarize, we found that the early stress-induced release of MMP-9 and other neutrophilassociated proteins was negatively associated with the cortisol response. In vitro studies further indicated that glucocorticoids were capable of suppressing the release of MMP-9 from neutrophils. Patients who were susceptible to stress-induced release of MMP-9 exhibited shorter leukocyte telomere length (as an indicator of cardiovascular aging) and larger burden of subclinical carotid 
atherosclerosis. The susceptibility to stress-induced release of MMP-9 may thus have impact on disease phenotype. Stress tests can be useful to identify CAD patients in need of novel prevention and treatment strategies.

\section{Role of funding sources}

This study was funded by the Swedish Research Council (K2012-64X-21175-04-3, K2015-65X-1459913-3) and the Swedish Heart-Lung Foundation (20120608). The funders did not have any role in designing, analyzing, writing, or making any decision about this article.

\section{Conflict of Interest}

The authors declare no conflicts of interest.

\section{References:}

Bellosta, S., D. Via, M. Canavesi, P. Pfister, R. Fumagalli, R. Paoletti and F. Bernini (1998). "HMG-CoA reductase inhibitors reduce MMP-9 secretion by macrophages." Arterioscler Thromb Vasc Biol 18(11): 1671-1678.

Blankenberg, S., H. J. Rupprecht, O. Poirier, C. Bickel, M. Smieja, G. Hafner, J. Meyer, F. Cambien and L. Tiret (2003). "Plasma concentrations and genetic variation of matrix metalloproteinase 9 and prognosis of patients with cardiovascular disease." Circulation 107(12): 1579-1585.

Brydon, L., Strike, P. C., Bhattacharyya, M. R., Whitehead, D. L., McEwan, J., Zachary, I., \& Steptoe, A (2010). "Hostility and physiological responses to laboratory stress in acute coronary syndrome patients." Journal of Psychosomatic Research 68(2): 109-116. 
Chakrabarti, S. and K. D. Patel (2005). "Regulation of matrix metalloproteinase-9 release from IL-8stimulated human neutrophils." J Leukoc Biol 78(1): 279-288.

Cook, W. W. and D. M. Medley (1954). "Proposed hostility and Pharisaic-virtue scales for the MMPI." Journal of Applied Psychology 38(6): 414-418.

Davidson, K. W., J. E. Schwartz, S. A. Kirkland, E. Mostofsky, D. Fink, D. Guernsey and D. Shimbo (2009). "Relation of inflammation to depression and incident coronary heart disease (from the Canadian Nova Scotia Health Survey [NSHS95] Prospective Population Study)." Am J Cardiol 103(6): 755-761.

Della Bona, R., M. T. Cardillo, M. Leo, G. Biasillo, M. Gustapane, F. Trotta and L. M. Biasucci (2013). "Polymorphonuclear neutrophils and instability of the atherosclerotic plaque: a causative role?" Inflamm Res 62(6): 537-550.

Domenici, E., D. R. Wille, F. Tozzi, I. Prokopenko, S. Miller, A. McKeown, C. Brittain, D. Rujescu, I. Giegling, C. W. Turck, F. Holsboer, E. T. Bullmore, L. Middleton, E. Merlo-Pich, R. C. Alexander and P. Muglia (2010). "Plasma protein biomarkers for depression and schizophrenia by multi analyte profiling of case-control collections." PLoS One 5(2): e9166.

Edmondson, D., J. D. Newman, W. Whang and K. W. Davidson (2013). "Emotional triggers in myocardial infarction: do they matter?" Eur Heart J 34(4): 300-306.

Ellard, D. R., P. C. Castle and R. Mian (2001). "The effect of a short-term mental stressor on neutrophil activation." Int J Psychophysiol 41(1): 93-100.

Fagundes, C. P., R. Glaser, B. S. Hwang, W. B. Malarkey and J. K. Kiecolt-Glaser (2013). "Depressive symptoms enhance stress-induced inflammatory responses." Brain Behav Immun 31: 172-176. Fernandez Machulsky, N., J. Gagliardi, B. Fabre, V. Miksztowicz, M. Lombardo, A. Garcia Escudero, G. Gigena, F. Blanco, R. J. Gelpi, L. Schreier, Y. Gidron and G. Berg (2016). "Matrix metalloproteinases and psychosocial factors in acute coronary syndrome patients." Psychoneuroendocrinology 63: 102108. 
Fyhrquist, F., O. Saijonmaa and T. Strandberg (2013). "The roles of senescence and telomere shortening in cardiovascular disease." Nat Rev Cardiol 10(5): 274-283.

Garvin, P., L. Jonasson, L. Nilsson, M. Falk and M. Kristenson (2015). "Plasma Matrix

Metalloproteinase-9 Levels Predict First-Time Coronary Heart Disease: An 8-Year Follow-Up of a Community-Based Middle Aged Population." PLoS One 10(9): e0138290.

Garvin, P., L. Nilsson, J. Carstensen, L. Jonasson and M. Kristenson (2009). "Plasma levels of matrix metalloproteinase-9 are independently associated with psychosocial factors in a middle-aged normal population." Psychosom Med 71(3): 292-300.

Heidt, T., H. B. Sager, G. Courties, P. Dutta, Y. Iwamoto, A. Zaltsman, C. von Zur Muhlen, C. Bode, G. L. Fricchione, J. Denninger, C. P. Lin, C. Vinegoni, P. Libby, F. K. Swirski, R. Weissleder and M. Nahrendorf (2014). "Chronic variable stress activates hematopoietic stem cells." Nat Med 20(7): 754758.

Hellberg, L., U. K. Samavedam, K. Holdorf, M. Hansel, A. Recke, T. Beckmann, K. Steinhorst, W. H. Boehncke, T. Kirchner, N. Mockel, W. Solbach, D. Zillikens, E. Schmidt, R. J. Ludwig and T. Laskay (2013). "Methylprednisolone blocks autoantibody-induced tissue damage in experimental models of bullous pemphigoid and epidermolysis bullosa acquisita through inhibition of neutrophil activation." J Invest Dermatol 133(10): 2390-2399.

Hellhammer, D. H., S. Wust and B. M. Kudielka (2009). "Salivary cortisol as a biomarker in stress research." Psychoneuroendocrinology 34(2): 163-171.

Hirsch, G., A. Lavoie-Lamoureux, G. Beauchamp and J. P. Lavoie (2012). "Neutrophils are not less sensitive than other blood leukocytes to the genomic effects of glucocorticoids." PLoS One 7(9): e44606.

Hua, Y., J. Xue, F. Sun, L. Zhu and M. Xie (2009). "Aspirin inhibits MMP-2 and MMP-9 expressions and activities through upregulation of PPARalpha/gamma and TIMP gene expressions in ox-LDLstimulated macrophages derived from human monocytes." Pharmacology 83(1): 18-25. 
Ionita, M. G., P. van den Borne, L. M. Catanzariti, F. L. Moll, J. P. de Vries, G. Pasterkamp, A. Vink and D. P. de Kleijn (2010). "High neutrophil numbers in human carotid atherosclerotic plaques are associated with characteristics of rupture-prone lesions." Arterioscler Thromb Vasc Biol 30(9): 18421848.

Jönsson, S., A. Lundberg, H. Kalvegren, I. Bergstrom, A. Szymanowski and L. Jonasson (2011). "Increased levels of leukocyte-derived MMP-9 in patients with stable angina pectoris." PLoS One 6(4): e19340.

Keresztes, M., Horváth, T., Ocsovszki, I., Földesi, I., Serfőző, G., Boda, K., \& Ungi, I (2013). " ACTH- and Cortisol-Associated Neutrophil Modulation in Coronary Artery Disease Patients Undergoing Stent Implantation." PLoS ONE 8(8): e71902.

Ketelhuth, D. F. and M. Back (2011). "The role of matrix metalloproteinases in atherothrombosis." Curr Atheroscler Rep 13(2): 162-169.

Kristenson, M., A. G. Olsson and Z. Kucinskiene (2005). "Good self-rated health is related to psychosocial resources and a strong cortisol response to acute stress: the LiVicordia study of middleaged men." Int J Behav Med 12(3): 153-160.

Kuebler, U., C. Zuccarella-Hackl, A. Arpagaus, J. M. Wolf, F. Farahmand, R. von Kanel, U. Ehlert and P. H. Wirtz (2015). "Stress-induced modulation of NF-kappaB activation, inflammation-associated gene expression, and cytokine levels in blood of healthy men." Brain Behav Immun 46: 87-95.

Kunz-Ebrecht, S. R., V. Mohamed-Ali, P. J. Feldman, C. Kirschbaum and A. Steptoe (2003). "Cortisol responses to mild psychological stress are inversely associated with proinflammatory cytokines." Brain Behav Immun 17(5): 373-383.

Lagraauw, H. M., J. Kuiper and I. Bot (2015). "Acute and chronic psychological stress as risk factors for cardiovascular disease: Insights gained from epidemiological, clinical and experimental studies." Brain Behav Immun 50: 18-30. 
Lin, J., E. Epel, J. Cheon, C. Kroenke, E. Sinclair, M. Bigos, O. Wolkowitz, S. Mellon and E. Blackburn (2010). "Analyses and comparisons of telomerase activity and telomere length in human T and B cells: insights for epidemiology of telomere maintenance." J Immunol Methods 352(1-2): 71-80. Liu, L., Y. X. Wang, J. Zhou, F. Long, H. W. Sun, Y. Liu, Y. Z. Chen and C. L. Jiang (2005). "Rapid nongenomic inhibitory effects of glucocorticoids on human neutrophil degranulation." Inflamm Res 54(1): 37-41.

McInnis, C. M., D. Wang, D. Gianferante, L. Hanlin, X. Chen, M. V. Thoma and N. Rohleder (2015). "Response and habituation of pro- and anti-inflammatory gene expression to repeated acute stress." Brain Behav Immun 46: 237-248.

Mian, R., G. Shelton-Rayner, B. Harkin and P. Williams (2003). "Observing a fictitious stressful event: haematological changes, including circulating leukocyte activation." Stress 6(1): 41-47.

Nabi, H., A. Singh-Manoux, M. Shipley, D. Gimeno, M. G. Marmot and M. Kivimaki (2008). "Do psychological factors affect inflammation and incident coronary heart disease: the Whitehall II Study." Arterioscler Thromb Vasc Biol 28(7): 1398-1406.

Newby, A. C. (2015). "Metalloproteinases promote plaque rupture and myocardial infarction: A persuasive concept waiting for clinical translation." Matrix Biol 44-46: 157-166.

Nijm, J., M. Kristenson, A. G. Olsson and L. Jonasson (2007). "Impaired cortisol response to acute stressors in patients with coronary disease. Implications for inflammatory activity." J Intern Med 262(3): 375-384.

Pearlin, L. I. (1978). "The structure of coping." Journal of Health and Social Behavior 19(1): 2-21. Radloff, L. (1977). "The CES-D Scale: A Self-Report Depression Scale for Research in the General Population." Applied Psychological Measurement 1(3): 385-401.

Rohleder, N. (2014). "Stimulation of systemic low-grade inflammation by psychosocial stress." Psychosom Med 76(3): 181-189. 
Schleimer, R. P., H. S. Freeland, S. P. Peters, K. E. Brown and C. P. Derse (1989). "An assessment of the effects of glucocorticoids on degranulation, chemotaxis, binding to vascular endothelium and formation of leukotriene B4 by purified human neutrophils." J Pharmacol Exp Ther 250(2): 598-605. Silvestre-Roig, C., M. P. de Winther, C. Weber, M. J. Daemen, E. Lutgens and O. Soehnlein (2014). "Atherosclerotic plaque destabilization: mechanisms, models, and therapeutic strategies." Circ Res 114(1): 214-226.

Soehnlein, O. (2012). "Multiple roles for neutrophils in atherosclerosis." Circ Res 110(6): 875-888. Steptoe, A., M. Hamer and Y. Chida (2007). "The effects of acute psychological stress on circulating inflammatory factors in humans: a review and meta-analysis." Brain Behav Immun 21(7): 901-912. Steptoe, A., G. Willemsen, N. Owen, L. Flower and V. Mohamed-Ali (2001). "Acute mental stress elicits delayed increases in circulating inflammatory cytokine levels." Clin Sci (Lond) 101(2): 185-192. Strike, P. C., K. Magid, D. L. Whitehead, L. Brydon, M. R. Bhattacharyya and A. Steptoe (2006). "Pathophysiological processes underlying emotional triggering of acute cardiac events." Proc Natl Acad Sci U S A 103(11): 4322-4327.

Szymanowski, A., J. Nijm, M. Kristenson and L. Jonasson (2011). "Elevated levels of circulating matrix metalloproteinase-9 are associated with a dysregulated cortisol rhythm--A case-control study of coronary artery disease." Psychoneuroendocrinology 36(1): 139-143.

Tomiyama, A. J., A. O'Donovan, J. Lin, E. Puterman, A. Lazaro, J. Chan, F. S. Dhabhar, O. Wolkowitz, C. Kirschbaum, E. Blackburn and E. Epel (2012). "Does cellular aging relate to patterns of allostasis? An examination of basal and stress reactive HPA axis activity and telomere length." Physiol Behav 106(1): 40-45.

Touboul, P. J., M. G. Hennerici, S. Meairs, H. Adams, P. Amarenco, N. Bornstein, L. Csiba, M. Desvarieux, S. Ebrahim, R. Hernandez Hernandez, M. Jaff, S. Kownator, T. Naqvi, P. Prati, T. Rundek, M. Sitzer, U. Schminke, J. C. Tardif, A. Taylor, E. Vicaut and K. S. Woo (2012). "Mannheim carotid intima-media thickness and plaque consensus (2004-2006-2011). An update on behalf of the advisory board of the $3 \mathrm{rd}$, 4th and 5th watching the risk symposia, at the 13th, 15th and 20th European Stroke 
Lundberg $A K$

Conferences, Mannheim, Germany, 2004, Brussels, Belgium, 2006, and Hamburg, Germany, 2011." Cerebrovasc Dis 34(4): 290-296.

Wikgren, M., M. Maripuu, T. Karlsson, K. Nordfjall, J. Bergdahl, J. Hultdin, J. Del-Favero, G. Roos, L. G. Nilsson, R. Adolfsson and K. F. Norrback (2012). "Short telomeres in depression and the general population are associated with a hypocortisolemic state." Biol Psychiatry 71(4): 294-300.

Wolf, J. M., N. Rohleder, A. Bierhaus, P. P. Nawroth and C. Kirschbaum (2009). "Determinants of the NF-kappaB response to acute psychosocial stress in humans." Brain Behav Immun 23(6): 742-749. 
Table 1. Characteristics of the study population. Values are shown as median (inter-quartile range), if nothing else is stated.

\section{Subjects $(n=64)$}

\section{Demographic and clinical measurements}

Female, $\mathrm{n}(\%)$

$10(16)$

Age, years

$66(61-72)$

Body mass index, $\mathrm{kg} / \mathrm{m}^{2}$

$27(25-30)$

Diabetes, $\mathrm{n}(\%)$

$11(17)$

Smoking, $\mathrm{n}(\%)$

$5(7.8)$

Myocardial infarction as index event, $\mathrm{n}(\%)$

$52(81)$

Coronary angiography, 0/1/2/3-vessel disease ${ }^{a}, n(\%)$

$3(5) / 20(31) / 22(34) / 19(30)$

\section{Cardiovascular medication}

Statin, $n(\%)$

$63(98)$

Dual anti-platelet therapy ${ }^{\mathrm{b}}, \mathrm{n}(\%)$

$61(95)$

$\beta$-blockers, n (\%)

$49(77)$

ACE-I/ARB, \% ${ }^{c}, \mathrm{n}(\%)$

$46(72)$

Calcium channel blockers, $\mathrm{n}(\%)$

$21(33)$

\section{Laboratory measurements}

Total cholesterol, $\mathrm{mmol} / \mathrm{L}$

$3.9(3.3-4.2)$

LDL cholesterol, $\mathrm{mmol} / \mathrm{L}$

HDL cholesterol, $\mathrm{mmol} / \mathrm{L}$

$1.2(1.0-1.3)$

Triglycerides, $\mathrm{mmol} / \mathrm{L}$

IL-6, pg/mL

$2.6(1.7-3.8)$

Leukocytes, cells $\times 10^{3} / \mu$ l

$6.5(5.5-7.5)$ 


\begin{tabular}{ll}
\hline Neutrophils, cells $\times 10^{3} / \mu l$ & $3.5(2.1-4.3)$ \\
\hline Leukocyte telomere length & $0.83(0.76-0.90)$ \\
\hline Psychological measurements $^{\mathrm{d}}$ & $8.9(3.3-15)$ \\
\hline Depressive symptoms & \\
\hline Cynical hostility & \\
\hline Coping ability & $29(23-33)$ \\
\hline Carotid ultrasound measurements & $24(21-26)$ \\
\hline IMT, left, mm & $0.80(0.65-0.90)$ \\
\hline IMT, right, mm & $0.80(0.70-0.95)$ \\
\hline Presence of plaques, left, $\mathrm{n}(\%)$ & $44(69)$ \\
\hline Presence of plaques, right, $\mathrm{n}(\%)$ & $52(81)$ \\
\hline
\end{tabular}

${ }^{a}=$ number of significantly stenosed vessels

${ }^{\mathrm{b}}=$ low-dose aspirin combined with $\mathrm{P}^{2} \mathrm{Y}_{12}$ antagonists (clopidogrel or ticagrelor)

c = angiotensin converting enzyme inhibitors/angiotensin receptor blockers

d $=$ scores represent mean values derived from 3 occasion

e $=$ Cronbach's alpha 0.86

${ }^{f}=$ Cronbach's alpha 0.85

$\mathrm{g}=$ Cronbach's alpha 0.75 
Table 2. Cardiovascular response to the laboratory stress test. Values indicate heart rate (beats per minute), systolic and diastolic blood pressure (BP, $\mathrm{mm} \mathrm{Hg}$ ) before test (baseline), peak levels at the end of stressor 1 and 2, respectively, and 20 min after the conclusion of stressor 2 (post-stress). Data are presented as median (inter-quartile range).

\begin{tabular}{llllll}
\hline & Baseline & Stressor 1 & Stressor 2 & Post-stress & $\mathbf{p}^{\mathbf{a}}$ \\
\hline Heart rate & $59(53-64)$ & $65(59-64)^{\dagger}$ & $66(57-75)^{\dagger}$ & $58(52-63)^{\ddagger}$ & $<0.001$ \\
Systolic BP & $140(128-150)$ & $156(138-171)^{\dagger}$ & $155(141-168)^{\dagger}$ & $137(123-150)^{\ddagger}$ & $<0.001$ \\
Diastolic BP & $79(73-85)$ & $87(79-97)^{\dagger}$ & $87(79-97)^{\dagger}$ & $79(72-85)^{\ddagger}$ & $<0.001$ \\
\hline
\end{tabular}

a $\mathrm{p}$ for trend (Friedman's test), for changes between two measurements (Wilcoxon signed-ranks test): ${ }^{\dagger} p<0.001$ vs baseline, ${ }^{\ddagger} p<0.001$ vs stressor 2 . 
Table 3. Correlations between log-transformed levels $(\mathrm{ng} / \mathrm{mL}$ ) of MMP-9, MMP-8, MPO, neutrophils counts and cortisol at baseline and percentage changes of MMP-9, MMP-8, MPO and cortisol after stress.

\begin{tabular}{|c|c|c|c|c|c|}
\hline & & MMP-9 & MMP-8 & MPO & Neutrophils \\
\hline & & serum & serum & serum & cells/ $\mu \mathrm{l}$ \\
\hline MMP-9 & serum & - & - & - & \\
\hline MMP-8 & serum & $0.759 * * *$ & - & - & \\
\hline MPO & serum & $0.594 * * *$ & $0.643^{* * *}$ & - & \\
\hline Neutrophils & cells/ $\mu l$ & $0.410 * *$ & $0.304^{* *}$ & $0.379 * * *$ & - \\
\hline \multirow[t]{2}{*}{ Cortisol } & $\mathrm{nmol} / \mathrm{L}$ & -0.151 & -0.062 & -0.110 & -0.025 \\
\hline & & $\%$ change & $\%$ change & $\%$ change & \\
\hline MMP-9 & $\%$ change & - & - & - & \\
\hline MMP-8 & $\%$ change & $0.802 * *$ & - & - & \\
\hline MPO & $\%$ change & $0.562 * *$ & $0.677^{* *}$ & - & \\
\hline Cortisol & $\%$ change & $-0.380 * *$ & $-0.299 *$ & $-0.386 * *$ & \\
\hline
\end{tabular}

Values are given as Pearson's correlation coefficients, ${ }^{*} p<0.05,{ }^{* *} p<0.01,{ }^{* * *} p<0.001$ 
Table 4. Selected characteristics, including laboratory variables, psychological measurements and cardiovascular reactivity, of CAD patients divided into tertiles; lower tertile (T1), mid-tertile (T2) and upper tertile (T3), depending on their percentage change in MMP-9. Data are presented as median (inter-quartile range).

\begin{tabular}{|c|c|c|c|c|c|}
\hline \multicolumn{3}{|c|}{ T1 $(n=22)$} & $T 2(n=22)$ & T3 $(n=22)$ & $\mathbf{P}^{d}$ \\
\hline \multicolumn{2}{|c|}{ MMP-8, change $\%$} & $-13(-25-(-6.0))$ & $-5.8(-13-5.8)^{*}$ & $35(21-72)^{\#,+}$ & $<0.001$ \\
\hline \multicolumn{2}{|c|}{ MPO, change $\%$} & $-4.7(-22-4.9)$ & $-3.9(-13-5.6)^{*}$ & $16(-1.0-53)^{\sharp, \dagger}$ & $<0.001$ \\
\hline \multicolumn{2}{|c|}{ Cortisol, change $\%$} & $-9.8(-29-60)$ & $-7.5(-26-15)$ & $-29(-39-(-8.0)) *$ & 0.02 \\
\hline \multicolumn{2}{|c|}{ IL- 6 baseline, $\mathrm{pg} / \mathrm{mL}$} & $2.2(1.4-2.8)$ & $1.8(1.3-3.2)$ & $2.5(1.8-4.1)^{*}$ & 0.05 \\
\hline \multicolumn{2}{|c|}{ Leukocyte telomere length } & $0.87(0.79-0.95)$ & $0.81(0.70-0.90)$ & $0.81(0.70-0.89)^{*}$ & 0.10 \\
\hline \multicolumn{6}{|c|}{ Psychological measurements ${ }^{\mathrm{a}}$} \\
\hline \multicolumn{2}{|c|}{ Depressive symptoms } & $8.0(3.0-14)$ & $3.0(1.3-11)$ & $8.0(3.0-16)$ & 0.35 \\
\hline \multicolumn{2}{|c|}{ Cynical hostility } & $27(21-36)$ & $27(19-32)$ & $29(21-36)$ & 0.69 \\
\hline \multicolumn{2}{|l|}{ Coping ability } & $24(20-25)$ & $24(22-26)$ & $25(21-26)$ & 0.44 \\
\hline \multicolumn{6}{|c|}{ Cardiovascular reactivity } \\
\hline \multirow[t]{3}{*}{ Heart rate ${ }^{\mathbf{b}}$} & Baseline & $58(55-64)$ & $59(51-69)$ & $59(53-65)$ & 0.89 \\
\hline & Stressor 2 & $66(57-70)$ & $69(57-80)$ & $65(59-72)$ & 0.71 \\
\hline & Post-stress & $57(52-63)$ & $58(50-67)$ & $58(52-65)$ & 0.91 \\
\hline \multirow[t]{3}{*}{ Systolic BP } & Baseline & $140(131-150)$ & $140(125-151)$ & $138(127-150)$ & 0.79 \\
\hline & Stressor 2 & $155(143-164)$ & $164(144-170)$ & $149(137-167)$ & 0.43 \\
\hline & Post-stress & 142 (121-149) & 139 (129-155) & $133(122-151)$ & 0.64 \\
\hline \multirow[t]{3}{*}{ Diastolic $\mathrm{BP}^{\mathrm{C}}$} & Baseline & $79(74-88)$ & $81(74-87)$ & $76(70-82)$ & 0.22 \\
\hline & Stressor 2 & 88 (80-99) & $93(83-100)$ & $84(77-91)$ & 0.08 \\
\hline & Post-stress & 80 (77-90) & $81(73-85)$ & $74(67-85)$ & 0.22 \\
\hline
\end{tabular}


Lundberg $A K$

${ }^{a}$ scores represent mean values derived from 3 occasions, ${ }^{b}$ beats per minute. ${ }^{c} \mathrm{~mm} \mathrm{Hg},{ }^{d} \mathrm{p}$ for trend (Kruskal-Wallis test), ${ }^{*} p<0.05$ vs T1, ${ }^{\#} p<0.001$ vs T1, ${ }^{\dagger} p<0.001$ vs T2, (Mann-Whitney U-test). 


\section{Figure captions}

Figure 1. Correlation between log transformed values of MMP-9 change and cortisol change during stress, both expressed as percentage change from baseline to 20 min after stress, Pearson's correlation coefficient, $r=-0.380, p=0.002$.

Figure 2. CAD patients divided into tertiles depending on their percentage change in MMP-9 after stress. Lower tertile (T1): $n=22$, mid-tertile (T2): $n=20$, upper tertile $(T 3): n=22$.

\section{Figure 3}

A) IL-8 induced the release of MMP-9 from isolated neutrophils in 10 individuals (5 CAD patients and 5 controls). All concentrations of dexamethasone; $10^{-6} \mathrm{M}\left(\mathrm{D}^{-6}\right), 10^{-7} \mathrm{M}\left(\mathrm{D}^{-7}\right), 10^{-8} \mathrm{M}\left(\mathrm{D}^{-8}\right)$ significantly reduced the IL- 8 induced release of MMP-9, $* * * p<0.001$. The bars show mean and SD.

B) IL-8 induced the release of MMP-8 from isolated neutrophils in 10 individuals (5 CAD patients and 5 controls). Dexamethasone $10^{-8} \mathrm{M}\left(\mathrm{D}^{-8}\right)$ significantly reduced the IL-8 induced release of MMP-9, while other concentrations, $10^{-6} \mathrm{M}\left(\mathrm{D}^{-6}\right)$ and $10^{-7} \mathrm{M}\left(\mathrm{D}^{-7}\right)$, tended to reduce it. The bars show mean and SD. 
Figure 1.

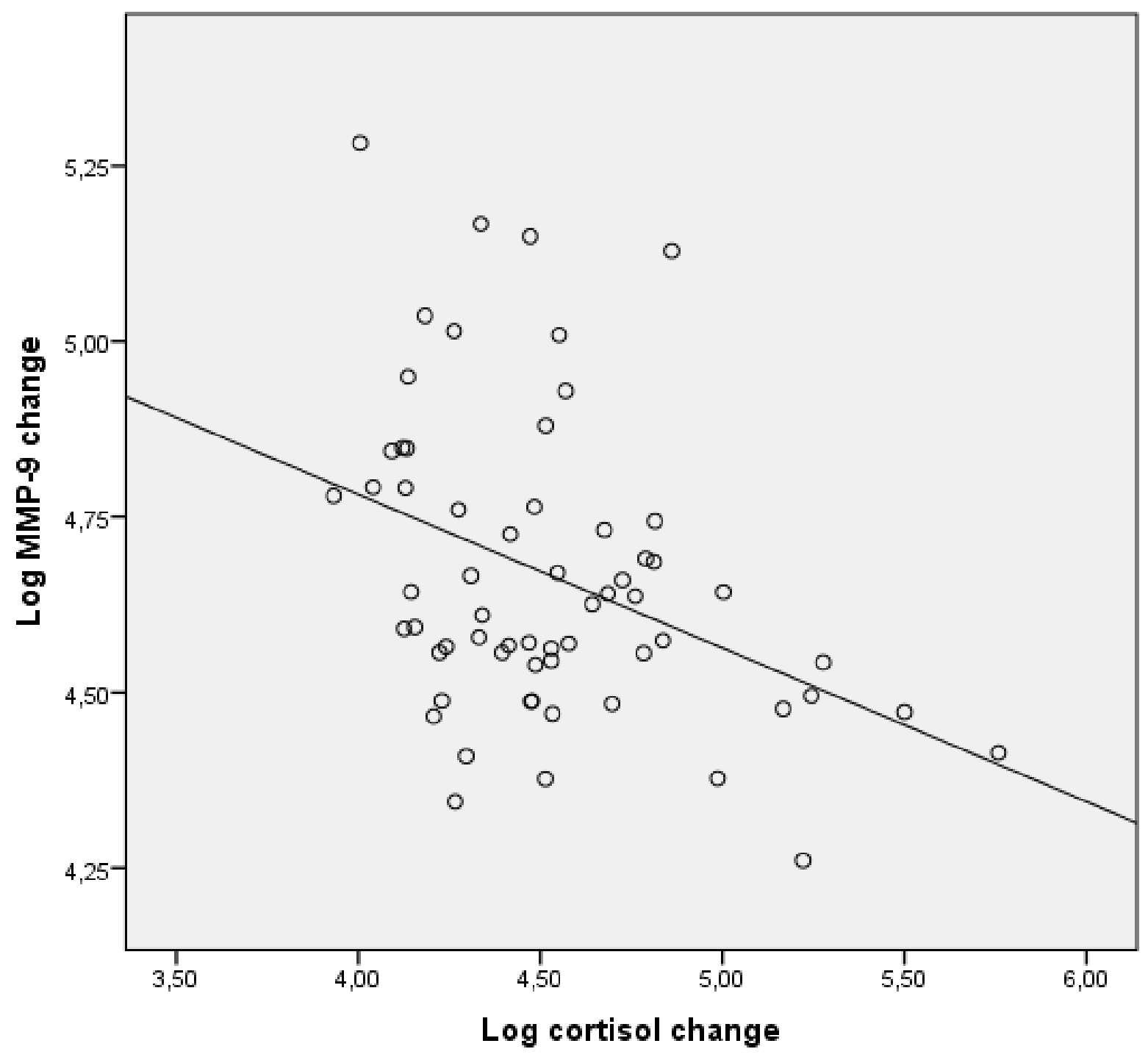


Figure 2.

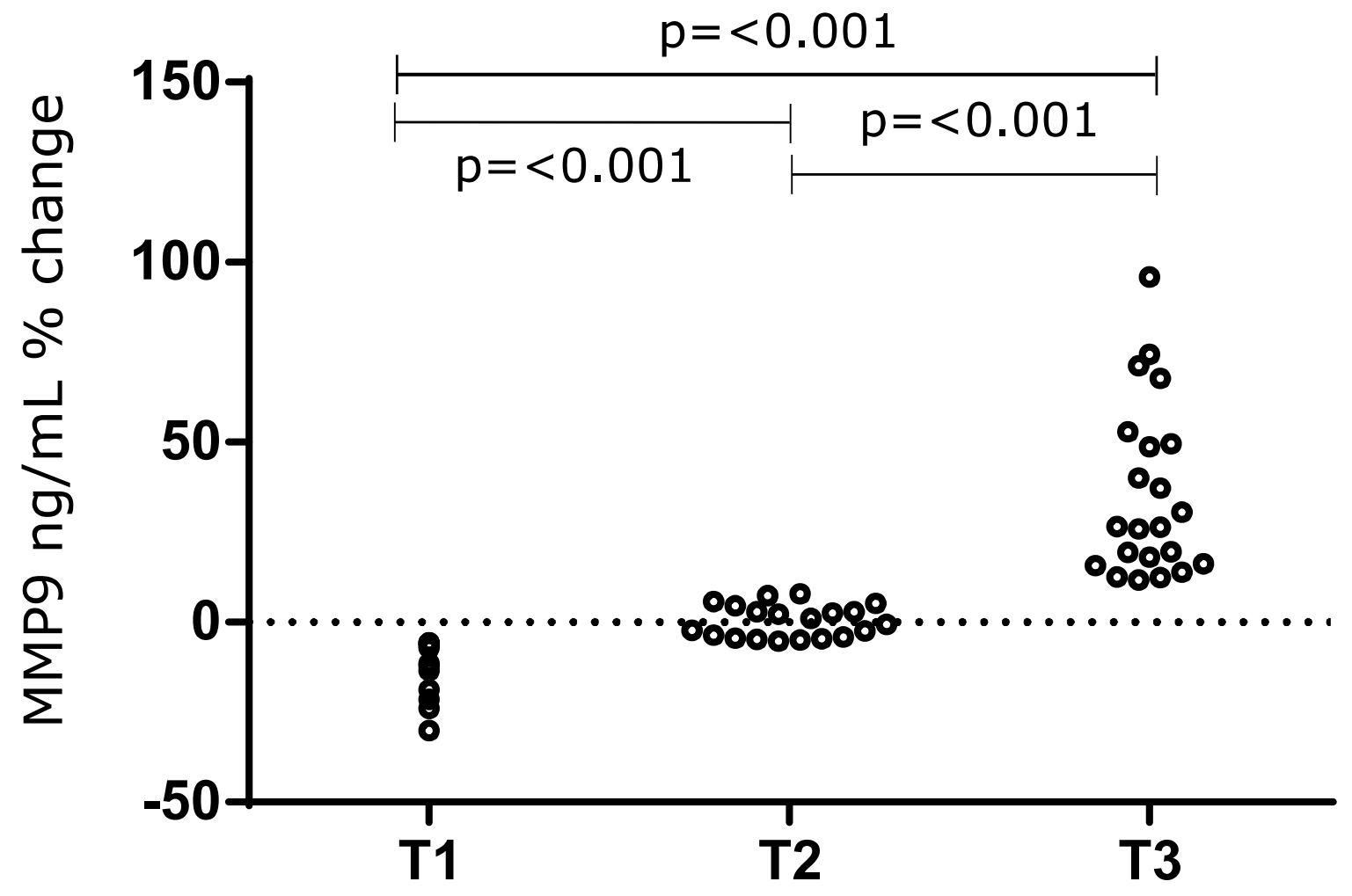


Figure 3.

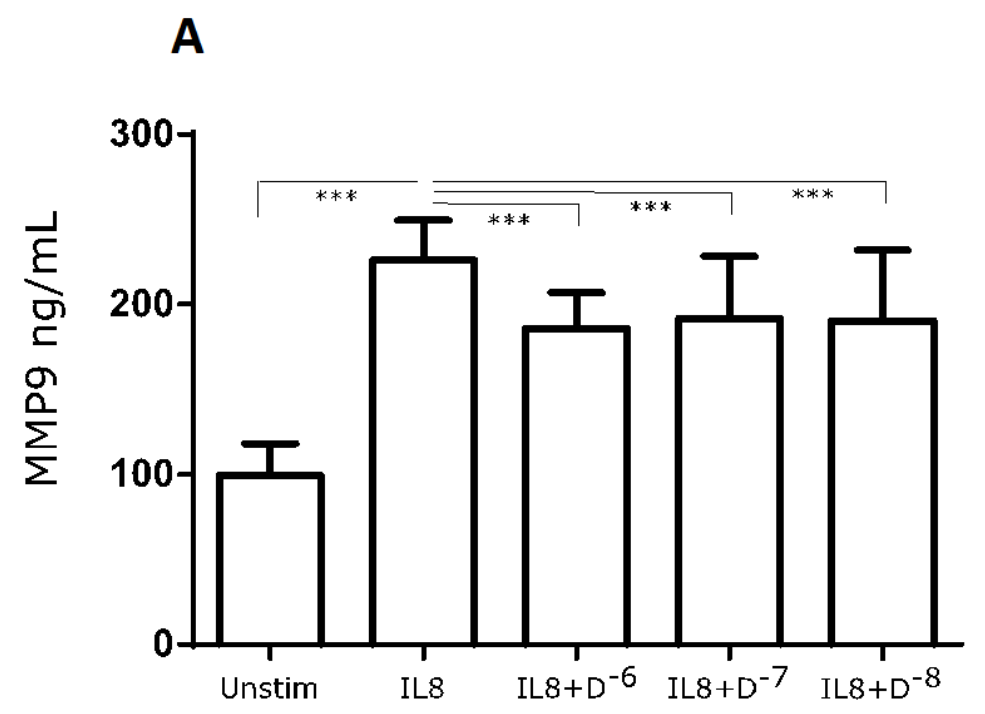

B

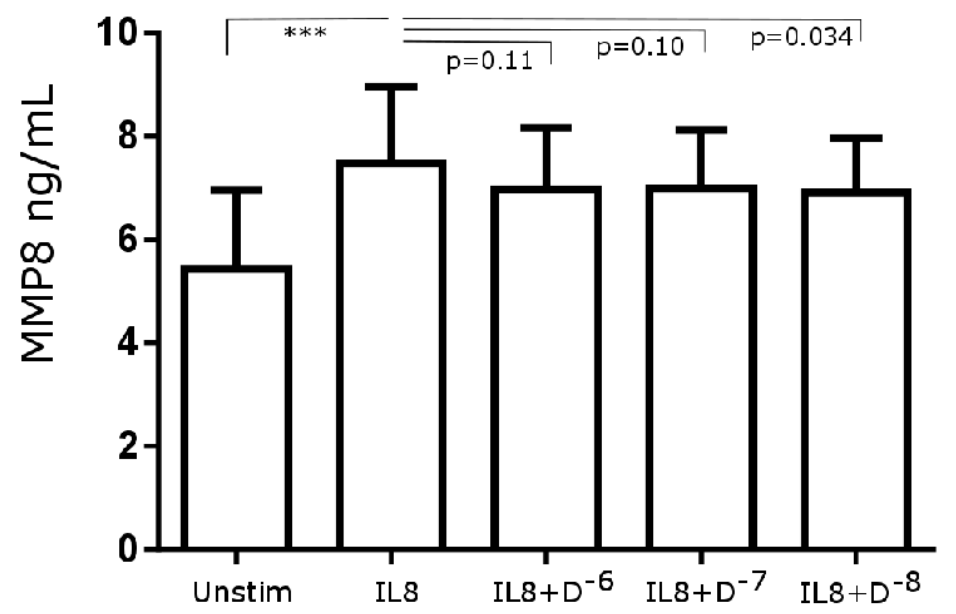

